\title{
Obtaining the lattice energy of the anthracene crystal by modern yet affordable first-principles methods
}

\author{
J. C. Sancho-García* \\ Departamento de Química Física, \\ Universidad de Alicante, E-03080 Alicante, Spain \\ and \\ Laboratory for Chemistry of Novel Materials, \\ University of Mons, B-7000 Mons, Belgium \\ J. Aragó and E. Ortí \\ Instituto de Ciencia Molecular, \\ Universidad de Valencia, E-46980 Valencia, Spain \\ Y. Olivier ${ }^{\dagger}$ \\ Laboratory for Chemistry of Novel Materials, \\ University of Mons, B-7000 Mons, Belgium
}

April 26, 2013

*E-mail: jc.sancho@ua.es

${ }^{\dagger}$ Also at Cavendish Laboratory, Optoelectronic group, University of Cambridge, Cambridge CB3 0HE, UK. 


\begin{abstract}
The non-covalent interactions in organic molecules are known to drive their self-assembly to form molecular crystals. We compare, in the case of anthracene and against experimental (electronic-only) sublimation energy, how modern quantum-chemical methods are able to calculate this cohesive energy taking into account all the interactions between occurring dimers in both first- and second-shells. These include both $O\left(N^{6}\right)$ - and $O\left(N^{5}\right)$-scaling methods, LPNO-pCCSD and SCS-MP2, respectively, as well as the most modern family of conceived density functionals: double-hybrid expressions in several variants (B2-PLYP, mPW2-PLYP, PWPB95) with customized dispersion corrections (-D3 and -NL). All-in-all, it is shown that these methods behave very accurately producing errors in the $1-2 \mathrm{~kJ} / \mathrm{mol}$ range with respect to the experimental value taken into account the experimental uncertainty. These methods are thus confirmed as excellent tools for studying all kind of interactions in chemical systems.
\end{abstract}




\section{Introduction}

Quantum and Computational Chemistry fields need more than ever to achieve a balanced description of all kind of systems, be they organic, inorganic or hybrid, and interactions, be they covalent or non-covalent, keeping at the same time the pervasive compromise needed between accuracy and cost to address nanotechnological or biological related issues. This is so because the bottom-up approach, lately followed to design new materials with targeted properties, new molecular nanoarchitectures or host-guest systems, or to study key biomolecules and their associated processes, can not accept large errors at the nanoscale, the first step in the size hierarchy, without affecting too much the specific properties at the mesoscale, where atomistic-like pictures are normally used to establish the link between both extremes of nature (micro- and macroscopic descriptions). Thus, working at the nanoscale demands the most of theoretical methods, which thus represents a real challenge since all quantum electronic effects truly manifest in these small dimensions and universally affect all systems.

As a matter of illustration, if one wants to study the key processes $[1,2]$ taking place within new organic-based electronic devices (ranging from fieldeffect transistors to clean energy devices or display technologies [3]) made from oligoacenes samples, it is needed to accurately describe any expected change in the self-assembly of the molecules driven by the underlying subtle forces between adjacent molecules (i.e. intermolecular interactions [4-6]). Despite the weakness of these forces, they really matter since any chemical functionalization of the molecular backbone might alter the mode of packing [7-9] and thus all the intended properties. On the other hand, polymorphism is a common issue affecting drug discoveries, crystal engineering or 
final composition of samples, demanding also the greatest possible accuracy to energetically rank the competing structures, which in many cases differ by just few $\mathrm{kJ} / \mathrm{mol}$ [10-17]. In this context, it is easy to understand why the clasically used metric known as "chemical accuracy" (deviation of \pm 1 $\mathrm{kcal} / \mathrm{mol}$ with respect to benchmark values) needs to be substituted here by the most stringent "calibration accuracy" (deviation of $\pm 1 \mathrm{~kJ} / \mathrm{mol}$ with respect to benchmark values).

However, achieving this accuracy is far to be a trivial task. First, highscaling quantum-chemical methods can give values close to it for the right reason $[18,19]$, but the price to be paid is of course the system size $(N)$. On the other hand, at the other extreme of the scale, methods based on Density Functional Theory (DFT) are very favorable in this respect, but they completely neglect long-range forces since, due to their specific construction, they can accurately describe electronic effects only at a limited neighborhood around the reference electronic position (the problem dubbed as near-sightedness of matter) and thus need specific corrections to become workable tools [20-37] able to deal with non-covalent interactions. Note that we have previously investigated [38] the performance of some low-cost DFT-based methods in the case of anthracene (see Figure 1), for which the crystalline structure and corresponding lattice energy were known. We will further investigate here if some of the most recently developed theoretical methods are able to achieve greater accuracy. This would allow to bracket their expected performance as well as to establish (if any) the existing relationships between accuracy and cost for these complicated interactions. 


\section{Theoretical methods}

\subsection{LPNO-pCCSD: $O\left(N^{6}\right)$}

Briefly speaking, this method is a parameterized (p) Coupled-Cluster Single and Double (CCSD) model within the Local Pair Natural Orbital (LPNO) approach, specifically devised to give accurate results with large basis sets. This is so because the virtual space expanded by large basis sets is much larger than the valence space, but the use of the corresponding natural orbitals largely accelerates the convergence of equations. Next, the introduction of some empirical parameters $(\alpha, \beta, \gamma)$ into the full CCSD equations, in fact into the contributions arising from the key term $\frac{1}{2} \hat{T}_{2}^{2}(\alpha$ and $\beta)$ and into the $\hat{T}_{1} \hat{T}_{2}$ term $(\gamma)$, leads to an accuracy close to that achieved by $\operatorname{CCSD}(\mathrm{T})$, the 'golden-standard' today, but at a non-negligible reduction of computational cost: $O\left(N^{6}\right)$ instead of $O\left(N^{7}\right)$. More details can be found in Refs. [39-43] while some of the specific applications to weak molecular interactions are gathered in Refs. [44-47].

\subsection{SCS-MP2 and SCS-S66-MP2: $O\left(N^{5}\right)$}

We start by recognizing the well-known fact that Møller-Plesset perturbation theory at second-order (MP2) is able to only partly capture the physics behind weak interactions, and it is thus considered as the pioneering yet simplest theoretical $a b$ initio method to be applied for it, although is is also wellknown how these interactions are systematically overestimated [48]. Briefly speaking again, starting from the uncorrelated Hartree-Fock (HF) energy, we can consider to scale differently the energy contributions arising from opposite- or same-spin interactions, in search of a greater accuracy than original MP2 does and actually close to that achieved by higher-order terms 
of the perturbation expansion, by doing:

$$
E^{\mathrm{MP} 2}=E^{\mathrm{HF}}+E_{c}^{\mathrm{PT} 2}=E^{\mathrm{HF}}+c_{\uparrow \downarrow} E_{c}^{\uparrow \downarrow}+\frac{1}{2} c_{\uparrow \uparrow} E_{c}^{\uparrow \uparrow}+\frac{1}{2} c_{\downarrow \downarrow} E_{c}^{\downarrow \downarrow}
$$

and try to find a set of optimal values for $c_{i j}$ against a respected and sound training set of accurate energies. This was initially implemented by S. Grimme [49] leading to the so-called Spin-Component-Scaled (SCS-) MP2 method. Admittedly, if we restrict the kind of interactions which are present in the training set, one can also obtain a set of parameters aiming at better describing those selected interactions. This has been recently done for the weak interactions contained in the S66 database [50] of weakly interacting complexes (SCS-S66-MP2). Note, however, that the general-purpose SCSMP2 behaves also successfully for these weak forces, as it exemplified by para-diiodobenzene [51] and rubrene [52] dimers, or paracyclophane derivatives [53], although perhaps tends to slightly underestimate these stabilizing weak effects [54]. We will apply here these two methods for the set of intermolecular interactions found in the anthracene crystal, in an attempt to further evaluate their reliability, and maked use of the default parameters defined for SCS-MP2 $\left(c_{\uparrow \downarrow}=6 / 5\right.$ and $\left.c_{\uparrow \uparrow}=c_{\downarrow \downarrow}=1 / 3\right)$, SCS-S66-MP2/def2$\operatorname{TZVP}\left(c_{\uparrow \downarrow}=0.04\right.$ and $\left.c_{\uparrow \uparrow}=c_{\downarrow \downarrow}=2.00\right)$, and SCS-S66-MP2/def2-QZVP $\left(c_{\uparrow \downarrow}=0.26\right.$ and $\left.c_{\uparrow \uparrow}=c_{\downarrow \downarrow}=1.56\right)$. Note how the parameters depend on the basis set chosen for the SCS-S66-MP2 variant.

\subsection{Double-hybrid density functionals: $O\left(N^{5}\right)$}

These methods are robustly rooted into the adiabatic connection framework [55-57], from which the hybrid functionals are also obtained after imposing some analytical path connecting the non-interacting particle system and the real interacting one [58-60], by introducing an additional depen- 
dence on the correlation energy obtained from perturbation theory up to second-order (PT2). Thus, together with the known mixture between exactlike exchange (EXX) energy, $E_{x}^{\mathrm{EXX}}$, and density functional exchange energy, $E_{x}[\rho]$, one adds now the mixture between the correlation energy obtained by perturbation theory, $E_{c}^{\mathrm{PT} 2}$, and the corresponding density functional correlation energy, $E_{c}[\rho]$, to get a general-purpose expression such as:

$$
E_{x c}^{\mathrm{DH}}=\mathrm{w}_{\mathrm{EXX}} E_{x}^{\mathrm{EXX}}+\left(1-\mathrm{w}_{\mathrm{EXX}}\right) E_{x}[\rho]+\mathrm{w}_{\mathrm{PT} 2} E_{c}^{\mathrm{PT} 2}+\left(1-\mathrm{w}_{\mathrm{PT} 2}\right) E_{c}[\rho],
$$

receiving correspondingly the name of double-hybrid (DH) density functionals. We will use the specific forms [61-63] called B2-PLYP ( $\mathrm{w}_{\mathrm{Exx}}=0.53$ and $\left.\mathrm{w}_{\mathrm{PT} 2}=0.27\right), \mathrm{mPW} 2-\mathrm{PLYP}\left(\mathrm{w}_{\mathrm{EXx}}=0.55\right.$ and $\left.\mathrm{w}_{\mathrm{PT} 2}=0.25\right)$, and PWPB95 $\left(\mathrm{w}_{\mathrm{EXX}}=0.50\right.$ and $\mathrm{w}_{\mathrm{PT} 2}=0.27$ for the $c_{\uparrow \downarrow}$ coefficient entering into the $E_{c}^{\mathrm{PT} 2}$ contribution, vanishing otherwise for same-spin contributions).

Final accuracy of DH functionals is believed to be determined by the value of $\mathrm{w}_{\mathrm{PT} 2}$ (i.e., indeed the $\mathrm{w}_{\mathrm{PT} 2} / \mathrm{w}_{\mathrm{EXX}}$ ratio) found in each case as a compromise. Without loss of generality, these DH forms can predict genuinely a bound dimer, contrarily to older DFT-based expressions. However, pairwise dispersion (2-body interactions) energy needs to be concomitantly added through a correction coined as -D2 [64,65] or -D3 [66,67] taking the form:

$$
E_{2-\text { body }} \approx E_{\mathrm{D} 3}=-\sum_{B>A}^{N} \sum_{n=6,8} s_{n} \frac{C_{n}^{A B}}{R_{A B}^{n}} f_{n}\left(R_{A B}, s_{r, n}\right),
$$

which runs over all atoms $A$ and $B$ of the system at their fixed (experimental here) positions. Note that: (i) $C_{n}^{A B}$ are the $n$ th-order interatomic dispersion coefficients; (ii) $f_{n}\left(R_{A B}, s_{r, n}\right)$ is a damping function peaking at the sum of van der Waals radii of $A$ and $B$ avoiding at the same singularities for short distances between atoms; (iii) $s_{n}$ and $s_{r, n}$ are the set of parameters efficiently 
coupling this term to the electronic energy given by eq. (2); (iv) the expression can be further simplified by keeping only the first term of the expansion, needing thus only the corresponding $s_{6}$ parameter, together with a systemindependent damping function or coefficients, to give the approach known as -D2; and (v) these corrections work independently of basis set issues and add at no extra computational cost. Furthermore, another way (not longer based on atom-to-atom pairwise interactions) to capture these long-range dispersion interactions relies on the instantaneous response of matter at a point $\mathbf{r}$ to a fluctuation of charge density at another point $\mathbf{r}^{\prime}$, which manifests in a truly nonlocal $(-\mathrm{NL})$ correction to the electronic energy in the way:

$$
E_{\mathrm{NL}}=\int d \mathbf{r} \rho(\mathbf{r})\left[\beta(b)+\frac{1}{2} \int d \mathbf{r}^{\prime} \rho\left(\mathbf{r}^{\prime}\right) \Phi\left(\mathbf{r}, \mathbf{r}^{\prime}\right)\right],
$$

needing to complementarily define the function $\Phi\left(\mathbf{r}, \mathbf{r}^{\prime}\right)$ coupling the densities at both points. We will use for it the specific modern construction VV10 [68] which can be added to any existing functional after defining the attenuation parameter $(b)$ entering into its formulation through $\beta$. This has been formerly done for pure and hybrid methods $[69,70]$ and recently extended by some of us to the case of double-hybrids [71]. Table 1 summarizes all the parameters used along this work for the dispersion corrections selected.

\subsection{Technical details}

The ORCA program [72] was used for all calculations, employing the sequence of def2-TZVP and def2-QZVP basis sets. Note that to reduce as much as possible the errors due to the basis set incompleteness, which are expected to largely affect intermolecular interaction energies, we always: (i) use for production purposes at the DFT level the very large def2-QZVP basis set; and (ii) extrapolate to the Complete Basis Set (CBS) limit the results of 
the ab initio calculations, for which a slower convergence pattern is known, using numerical techniques allowing to reach the asymptotic regime. The Basis Set Superposition Error (BSSE) is expected to be thus negligible in both cases and, consequently, the interaction energies are not counterpoise corrected. Note also that the counterpoise method to estimate the (possible) BSSE is believed to overestimate its effect, and some authors even propose to scale it down by half of its value [37]. The computational effort is significantly reduced in all the cases by making use of the 'resolution of the identity' (RI) [73] and the 'chain-of-spheres' (COSX) [74] algorithms, for Coulomb or exchange integrals, respectively, using for it the corresponding matching auxiliary basis sets [75]. The quadrature grids needed for numerical integration of density functionals are also increased with respect to defaults, which is strongly recommended for intermolecular interaction energies, as well as the corresponding thresholds for converging energies self-consistently. The MERCURY program [76] is employed for visualizing and manipulating crystal structures in three dimensions.

\section{Accessing cohesive energy from intermolec- ular interactions}

The lattice energy, actually the binding energy arising from all intermolecular interactions, is related to the sublimation enthalpy of the molecular crystal. However, thermal and vibrational effects need to be carefully taken into account before any comparison between experiments and theory can be made. Fortunately, this has been recently done for a set of organic solids of the most interest [77], starting from experimental data and correcting for temperature effects and solid vibrations, readily allowing to compare 
the estimates of approximate yet accurate methods. Therefore, in the case of interest here, the anthracene crystal, an (electronic-only) experimental lattice energy $\left(\Delta E_{\text {latt }}\right)$ of $100.6 \mathrm{~kJ} / \mathrm{mol}$ is obtained, although we also need to consider the average experimental error for sublimation energies $( \pm 4.9$ $\mathrm{kJ} / \mathrm{mol}$ ) [78] before assessing the results of any theoretical method.

We base our calculations on dimer interactions, for which the interaction energy is defined as $\Delta E_{i}=E_{\operatorname{dimer}(i)}-2 E_{\text {monomer }}$. The coordinates of all the anthracene dimers belonging to first and second coordination shells (see Figure 2) were taken from Ref. [79] and were not otherwise optimized. Note that: (i) previous and careful estimates of cohesive energy from dimer interactions (benzene [80]) were certainly successful; (ii) the convergence is believed to be attained since the consideration of additional shells is expected to lead to negligible (note the well-known $r^{-6}$ decay of these interactions) energy contributions; and (iii) the use of a nanoaggregate, instead of the set of all considered dimers, would add the problem of many-body interactions which is not still completely solved despite recent interesting advances in the right direction [81-83]. Finally, to get an estimate of the cohesive or lattice energy, one needs to multiply each association energy by the number $(m)$ of symmetry-related pairs, $-\Delta E_{l a t t}=m_{i} \sum_{i} \Delta E_{i}$, and then to divide the results by two as a result of the counting method, see Ref. [84]. Note how the final value of $\Delta E_{l a t t}$ is made positive to compare with experimental (electroniconly) sublimation energies.

The behavior of canonical MP2 is analyzed first as reference for further improvements. Whereas the result for $\Delta E_{\text {latt }}$ with the def2-TZVP basis set is affected of a large error $(158.3 \mathrm{~kJ} / \mathrm{mol})$, the use of def2-QZVP largely reduces 
it $(132.3 \mathrm{~kJ} / \mathrm{mol})$ and brings it closer to the CBS estimate $(123.9 \mathrm{~kJ} / \mathrm{mol})$ after applying an extrapolation technique using the well-known dependence $\left(n^{-3}\right.$, where $n$ is related to the largest angular moment of the basis functions for each considered basis set) of this correlation energy with respect to basis set size [85]. We employ now the LPNO-pCCSD method in two variants (LPNO-pCCSD1a, having $\alpha=-1$ and $\beta=\gamma=1$, and LPNOpCCSD1b, having $\alpha=\gamma=-1$ and $\beta=1$ ). We will refer in the following only to the LPNO-pCCSD1a results because both approaches lead to practically indistinguishable results. Using the def2-TZVP basis set drops a value of $114.4 \mathrm{~kJ} / \mathrm{mol}$ for $\Delta E_{\text {latt }}$, which may be hopefully improved considering the CBS limit. Then, taking into account that: (i) the $O\left(N^{6}\right)$ scaling of LPNOpCCSD1a precludes its application with basis sets larger than the def2-TZVP one, and (ii) basis set effects on correlation energy $\left(E_{c}\right)$ can be efficiently captured at the MP2 level; we approximate the final (nearly-converged) energies by:

$$
\begin{aligned}
E_{\mathrm{LPNO}-\mathrm{pCCSD} 1 \mathrm{a} / \mathrm{CBS}} & \approx E^{\mathrm{HF} / \mathrm{def} 2-\mathrm{QZVP}}+E_{c}^{\mathrm{MP} 2 / \mathrm{CBS}} \\
& +\left(E_{c}^{\mathrm{LPNO}-\mathrm{pCCSD} 1 \mathrm{a} / \mathrm{def} 2-\mathrm{TZVP}}-E_{c}^{\mathrm{MP} 2 / \mathrm{def} 2-\mathrm{TZVP}}\right)
\end{aligned}
$$

as it has been also done before with much success $[44,45]$. Note that we assume that: (i) HF energies are practically converged at the def2-QZVP level; and (ii) the residual difference between LPNO-pCCSD1a and MP2 correlation energies has a less marked dependence on basis sets than the MP2 correlation energy itself. We obtain now a value for $\Delta E_{\text {latt }}$ of $94.7 \mathrm{~kJ} / \mathrm{mol}$, which can be considered of large accuracy and close to the experimental estimate (see Figure 3). As a test of self-consistency of the extrapolation schemes investigated, if one substitutes now $E_{c}^{\mathrm{MP} 2 / \mathrm{CBS}}$ by $E_{c}^{\mathrm{MP} 2 / \operatorname{def} 2-\mathrm{QZVP}}$ in eq. (5) the value of $\Delta E_{\text {latt }}$ is only slightly affected $(95.3 \mathrm{~kJ} / \mathrm{mol})$ in the right direction. Other investigated approaches, for instance a slower $n^{-2.4}$ convergence 
for MP2-derived correlation energies [86], to estimate the CBS limit led to less accurate results and will be thus not shown.

The SCS-MP2 results are presented next: whereas Table 2 gathers the $\Delta E_{i}$ values corresponding to all dimer interactions, the resulting $\Delta E_{\text {latt }}$ energy is included in Figure 3 too. Note that we also extrapolate the correlation energy provided by def2-TZVP and def2-QZVP using the same function than before for the MP2 case. The accuracy of this scheme will be investigated elsewhere. As a matter of example, we focus on the $\Delta E_{i}$ energy of the $(\mathrm{a} / 2, \mathrm{~b} / 2,0)$ pair, see Figure 2, which actually drives the herringbone-like growing of anthracene molecules contributing the most to $\Delta E_{l a t t}$ in all cases, to see how its value consistently decreases upon larger basis sets: -34.0 , 29.8 , and $-28.2 \mathrm{~kJ} / \mathrm{mol}$ with the def2-TZVP, def2-QZVP, and at the CBS limit, respectively. We have also detected with not-so-large basis sets (i.e., def2-TZVP) how some interaction energies (admittedly small) become in this case positive $\left(\Delta E_{i}>0\right)$ and can thus perturb the final estimate of $\Delta E_{\text {latt }}$ resulting in a slight underbinding. This spurious numerical effect can be attributed again to the basis set incompleteness since, as it can be observed in Table 2, all the intermolecular interactions have the right sign once the CBS limit is achieved. The accuracy of the SCS-S66-MP2 variant is close to that of SCS-MP2 although some appreciations appear also to be in order: (i) the cohesive energy with the def2-TZVP (def2-QZVP), and the specific sameand opposite-spin scaling parameters for each basis set, is strongly (slightly) overestimated finding again that $\Delta E_{i}>0$ for some dimers, resulting in final values of 150.0 and $111.6 \mathrm{~kJ} / \mathrm{mol}$, respectively; (ii) we observe a more pronounced dependence of the results with basis set effects, since going from def2-TZVP to def2-QZVP the value of $\Delta E_{\text {latt }}$ is reduced by a rough $25 \%$ 
(20\% in the SCS-MP2 case); and (iii) since the scaling parameters depend on basis sets, and can thus partly mimic their incompleteness, we do not perform the corresponding extrapolation to the CBS limit.

The previously derived MP2 values can be also helpful to approximately interpret the results of the double-hybrid calculations. Actually, the B2PLYP method missing the dispersion correction provides a value of 34.6 $\mathrm{kJ} / \mathrm{mol}$, and thus severely underestimates the experimental value, which qualitatively correlates with the weight of the PT2-like term entering into its formulation $\left(\Delta E_{\text {latt }}^{\mathrm{B} 2-\mathrm{PLYP}} \approx \mathrm{W}_{\mathrm{PT} 2} \Delta E_{\text {latt }}^{\mathrm{MP} 2}\right)$. This underlines again the importance of the PT2-like term for double-hybrid calculations, since corresponding semilocal pure or hybrid functionals completely neglect these dispersion interactions and give thus unbound dimers. Note that in the case of mPW2-PLYP or PWPB95, the exchange functional was reparameterized to partially mimic long-range interactions, and thus they might behave slightly better (49.5 and $44.8 \mathrm{~kJ} / \mathrm{mol}$, respectively). Even if these values are admittedly affected of large errors, more than $50 \%$, they serve as a good starting point for adding the dispersion energy still missed and thus for further discussing the rest of results. One can easily see from Table 2 how accurate are now the predictions of all schemes used, for instance, with the B2-PLYP model, that is, the B2-PLYP-D2, B2-PLYP-D3, and the B2-PLYP-NL corrections. They predict values between $108-110 \mathrm{~kJ} / \mathrm{mol}$, which can be considered very close to the experimental estimate (see Figure 3), and indeed almost all the tested DH functionals provided very accurate values, including mPW2-PLYP-D2 (102.6 kJ/mol) and PWPB95-D3 (105.3 kJ/mol). Although the leading contributions to the cohesive energy mainly arise from the inner coordination sphere, interactions from the outer sphere account for $24-26 \%$ of its value 
and can be thus considered as significant. Note also that the slower convergence of the PT2-type term upon increasing basis sets, compared with the other functional terms, is not expected to modify significantly the results when a sufficiently large (e.g. def2-QZVP) basis set is used. However, we have also to recognize an error (around $\pm 1-2 \mathrm{~kJ} / \mathrm{mol}$ ) in these theoretical estimates arising from the positive energy values for the $(-\mathrm{a}, 0, \mathrm{c})$ and $(-\mathrm{a}, \mathrm{b}, \mathrm{c})$ dimers. Furthermore, we have also assessed the influence of longer range interactions through the dimer $(0,2 \mathrm{~b}, 0)$ belonging to the third-shell. The results obtained with B2-PLYP-D2, B2-PLYP-D3, and B2-PLYP-NL are about $-0.1 \mathrm{~kJ} / \mathrm{mol}$, between $150-250$ times smaller that for the corresponding 2nd-shell dimer $(0, \mathrm{~b}, 0)$, and can thus considered as negligible.

The -D3 scheme can be further corrected by accounting for the influence of three-body terms (i.e., the contributions for all atoms triples $A, B$, and $C$ ) by means of the following expression [87]:

$E_{3-\text { body }} \approx E^{\mathrm{ABC}}=\sum_{A>B>B}^{N} C_{9}^{A B C} \frac{\left(3 \cos \theta_{a} \cos \theta_{b} \cos \theta_{c}+1\right)}{\left(R_{A B} R_{B C} R_{A C}\right)^{3}} f_{n}\left(R_{A B}, R_{B C}, R_{A C}\right)$,

where $C_{9}^{A B C}$ is the corresponding coefficient (approximated by $C_{9}^{A B C}=$ $-\sqrt{C_{6}^{A B} C_{6}^{B C} C_{6}^{A C}}$ ) and $\theta_{i}$ are the internal angles of the triangle formed by the interatomic distances $\left(R_{A B} R_{B C} R_{A C}\right)$. Although this correction may be in principle applied to all schemes, we prefer to keep consistency with original developments and then to restrict it to the case of -D3. Note that we have evaluated in the past [38] this contribution for the nanoaggregate of anthracene molecules depicted in Figure 4, which corresponds to the positions of the molecules in the $a-b$ plane of the crystalline lattice (the most densely packed) and thus represents a trade-off between size and computational resources, to obtain a value of $7.2 \mathrm{~kJ} / \mathrm{mol}$. Note that this contribution 
is positive and thus correct the typical slight overbinding of the -D3-based approaches. The final B2-PLYP-D3 and PWPB95-D3 cohesive energies are now 103.3 and $98.1 \mathrm{~kJ} / \mathrm{mol}$, respectively, lying within the experimental accuracy. This reinforces the robustness of all tested dispersion corrections since, almost independently of the underlying exchange-correlation functional, they are able to largely overcome the underestimation found for the uncorrected expressions and can thus be used safely for related properties and systems using finite-model approaches.

Finally, we remind that previous estimates of lattice energies of anthracene crystals were mainly performed with $O\left(N^{3}\right)$ or $O\left(N^{4}\right)$ methods, employing different dispersion corrections and technical conditions, which makes difficult the direct comparison of values. Notwidthstanding this, and fixing the functional $\mathrm{PBE}$ in all cases, we mention values ranging from 51.6 to 135.5 $\mathrm{kJ} / \mathrm{mol}$. More specifically, results within PBE-lg (51.6 kJ/mol [88]), PBEXDM (96.3 kJmol [77]), PBE-D2 (104.3 kJ/mol [89] and $106.22 \mathrm{~kJ} / \mathrm{mol}$ [77]), and PBE-TS (130.5 kJ/mol [89] and $135.5 \mathrm{~kJ} / \mathrm{mol}$ [77]) schemes are reported in the literature, which indicates a strong influence on the results of the formerly employed dispersion corrections as well as their technicalities. As a double-check, note how B3LYP-D2 drops a value of $105.9 \mathrm{~kJ} / \mathrm{mol}$ [90] being thus close to PBE-D2, as it should be expected. If one fixes now the revised PBE (revPBE) functional [91], we had previously obtained values between 128-126 (112-108) kJ/mol when the variants revPBE-D3 and revPBE0-D3 (revPBE-NL and revPBE0-NL), respectively, were employed [38]. On the other hand, all the methods considered here, independently of their different origin and parameterization and once basis set incompleteness issues are solved, lead to values ranging between $96-110 \mathrm{~kJ} / \mathrm{mol}$ to be compared with 
an experimental estimate of $101 \pm 5 \mathrm{~kJ} / \mathrm{mol}$.

\section{Conclusions}

The interactions between weakly overlapping densities become a serious challenge for any modern quantum-chemical method as far as a decent compromise between accuracy and computational effort needs to be always achieved. Searching to shed light about this issue, we have systematically studied by means of modern theoretical methods the non-covalent interactions appearing in the case of the anthracene molecular crystal. These interactions are known to drive the final supramolecular arrangement of samples, the crystal growth preferred direction and the possible (if any) polymorphism of molecular materials. It was found that the LPNO-pCCSD1a (and related) method behaves sufficiently accurate for such applications, essentially capturing the physics of weak interactions, as well as the reference $\operatorname{CCSD}(\mathrm{T})$ method would expectedly do, but here at an order of magnitude (concerning computational cost and resources) lower. The same statement about the excellent performance of the SCS-MP2 modification of MP2 can be also made, with an additional decrease in cost by an order of magnitude, which might thus hopefully pave the way towards more and more applications within the field. The MP2 method could be soon considered as superseded, since apparently there is no reason to further exploit the SCS-based variants. Interestingly, the use of double-hybrid density functionals warrants to achieve the desired accuracy once the dispersion correction $(-\mathrm{D} 2,-\mathrm{D} 3$ or $-\mathrm{NL})$ is added, without being affected of any erratic performance or of a large variation of values after considering the different corrections. Therefore, we remain optimistic about the alleged reputation of these methods as efficient, robust and 
accurate for current research and associated challenges when dealing with non-covalent interactions between $\pi$-conjugated systems of large size.

\section{Acknowledgements}

Financial support by the "Ministerio de Economía y Competitividad" of Spain and the "European Regional Development Fund" through projects CTQ2011-27253, CTQ2012-31914, and Consolider-Ingenio CSD2007-00010 in Molecular Nanoscience, and by the "Generalitat Valenciana" (ISIC 2012/008) is acknowledged. The work in Mons is supported by the Belgian National Fund for Scientific Research (FNRS). Y.O. is a FNRS Post-doctoral Research Fellow. J.C.S.G. is a FNRS Visiting Professor.

\section{References}

[1] V. Coropceanu, J. Cornil, D. A. da Silva Filho, Y. Olivier, R. Silbey, and J. L. Bredás, Chem. Rev. 107, 926 (2007).

[2] J. L. Brédas, D. Beljonne, S. Coropceanu, and J. Cornil, Chem. Rev. 104, 4971 (2004).

[3] C. Wang, H. Dong, W. Hu, Y. Liu, and D. Zhu, Chem. Rev. 112, 2208 (2012).

[4] D. Käfer, M. El Helou, C. Gemel, and G. Witte, Cryst. Growth Des. 8, 3053 (2008).

[5] J. D. Dunitz and A. Gavezzotti, Chem. Soc. Rev. 38, 2622 (2009).

[6] J. F. Dobson and T. Gould, J. Phys.: Condens. Matter 24, 073201 (2012). 
[7] J. E. Anthony, Chem. Rev. 106, 5028 (2006).

[8] X. Chi, D. Li, H. Zhang, Y. Chen, V. García, C. García, and T. Siegrist, Org. Electron. 9, 234 (2008).

[9] G. Schweicher, N. Paquay, C. Amato, R. Resel, M. Koini, S. Talvy, J. Cornil, V. Lemaur, Y. Geerts, and G. Gbabode, Crystal Growth \& Design 11, 3663 (2011).

[10] S. L. Price, Adv. Drug Delivery Res. 56, 301 (2004).

[11] P. G. Karamertzanis, G. M. Day, G. W. a. Welch, J. Kendrick, F. J. J. Leusen, M. A. Neumann, and S. L. Price, J. Chem. Phys. 128, 244708 (2008).

[12] S. L. Price, Int. Rev. Phys. Chem. 27, 541 (2008).

[13] G. M. Day, T. G. Cooper, A. J. Cruz-Cabeza, K. E. Hejczyk, H. L. Ammon, S. X. M. Boerrigter, J. S. Tan, R. G. Della Valle, E. Vet, J. Jose, S. R. Gadre, G. R. Desiraju, T. S. Thakur, B. P. van Eijck, J. C. Facelli, V. E. Bazterra, M. B. Ferraro, D. W. M. Hofmann, M. A. Neumann, F. J. J. Leusen, J. Kendrick, S. L. Price, A. J. Misquita, P. G. Karamertzanis, G. W. A. Welch, H. A. Scheraga, Y. A. Amutova, M. U. Schmidt, J. van de Streek, A. K. Wolf, and B. Schweizer, Acta Cryst. B65, 107 (2009).

[14] C. Ambrosch-Draxl, D. Nabok, P. Puschnig, and C. Meisenbichler, New J. Phys. 11, 125010 (2009).

[15] J. Moellmann and S. Grimme, Phys. Chem. Chem. Phys. 12, 8500 (2010). 
[16] L. Maschio, B. Civalleri, P. Ugliengo, and A. Gavezzotti, J. Phys. Chem. A 115, 11179 (2011).

[17] S. Wen and G. J. O. Beran, J. Chem. Theory Comput. 8, 2698 (2012).

[18] J. Č́rny and P. Hobza, Phys. Chem. Chem. Phys. 9, 5291 (2007).

[19] K. E. Riley, M. Pitoňák, P. Jurečka, and P. Hobza, Chem. Rev. 110, $5023(2010)$.

[20] M. Dion, H. Rydberg, E. Schröder, D. C. Langreth, and B. I. Lundqvist, Phys. Rev. Lett. 92, 246401 (2004).

[21] O. von Lilienfeld, I. Tavernelli, U. Rothlisberger, and D. Sebastiani, Phys. Rev. Lett. 93, 153004 (2004).

[22] S. Grimme, J. Comput. Chem. 25, 1463 (2004).

[23] Y. Zhao and D. G. Truhlar, J. Phys. Chem. A. 109, 5656 (2004).

[24] M. Neumann and M.-A. Perrin, J. Phys. Chem. B 109, 15531 (2009).

[25] Y. Zhao and D. G. Truhlar, J. Chem. Theory Comput. 2, 364 (2006).

[26] A. D. Becke and E. R. Johnson, J. Chem. Phys. 124, 014104 (2006).

[27] A. D. Becke and E. R. Johnson, J. Chem. Phys. 127, 154108 (2007).

[28] S. Grimme, J. Antony, T. Schwabe, and C. Mück-Lichtenfeld, Org. Biomol. Chem. 5, 741 (2007).

[29] G. DiLabio, Chem. Phys. Lett. 455, 348 (2008).

[30] E. R. Johnson, I. D. Mackie, and G. A. DiLabio, J. Phys. Org. Chem. 22, 1127 (2009). 
[31] A. Tkatchenko and M. Scheffler, Phys. Rev. Lett. 102, 073005 (2009).

[32] D. Lu, Y. Li, D. Rocca, and G. Galli, Phys. Rev. Lett. 102, 206411 (2009).

[33] T. Schwabe, R. Huenerbein, and S. Grimme, Synlett 10, 1431 (2010).

[34] N. Marom, A. Tkatchenko, M. Rossi, V. V. Gobre, O. Hod, M. Scheffler, and L. Kronik, J. Chem. Theory Comput. 7, 3944 (2011).

[35] Y. Li, D. Lu, H.-V. Nguyen, and G. Galli, J. Phys. Chem. A 114, 1944 (2010).

[36] A. Otero-de-la-Roza and E. R. Johnson, J. Chem. Phys. 138, 054103 (2013).

[37] T. Risthaus and S. Grimme, J. Chem. Theory Comput. 9, 1580 (2013).

[38] J. C. Sancho-García and Y. Olivier, J. Chem. Phys. 137, 194311 (2012).

[39] F. Neese, F. Wennmohs, and A. Hansen, J. Chem. Phys. 130, 114108 (2009).

[40] F. Neese, A. Hansen, and D. G. Liakos, J. Chem. Phys. 131, 064103 (2009).

[41] L. M. J. Huntington and M. Noojien, J. Chem. Phys. 133, 184109 (2010).

[42] A. Hansen, D. G. Liakos, and F. Neese, J. Chem. Phys. 135, 214102 (2011).

[43] L. M. J. Huntington, A. Hansen, F. Neese, and M. Noojien, J. Chem. Phys. 136, 064101 (2012). 
[44] D. G. Liakos, A. Hansen, and F. Neese, J. Chem. Theory Comput. 7, 76 (2011).

[45] D. G. Liakos and F. Neese, J. Phys. Chem. A 116, 4801 (2012).

[46] T. Schwabe, J. Comput. Chem. 33, 2067 (2012).

[47] C. Riplinger and F. Neese, J. Chem. Phys. 138, 034106 (2013).

[48] D. Cremer, WIREs Comp. Mol. Sci. 1, 509 (2011).

[49] S. Grimme, J. Chem. Phys. 118, 9095 (2003).

[50] K. E. Riley, J. A. Platts, J. Rezáč, P. Hobza, J. Grant Hill, J. Phys. Chem. A 116, 4159 (2012).

[51] C. R. Taylor, P. J. Bygrave, J. N. Hart, N. L. Allan, and F. R. Manby, Phys. Chem. Chem. Phys. 14, 7739 (2012).

[52] J. C. Sancho-García and A. J. Pérez-Jiménez, J. Chem. Phys. 129, 024103 (2008).

[53] P. Hemberger, A. Bodi, C. Schon, M. Steinbauer, K. H. Fischer, C. Kaiser, and I. Fischer, Phys. Chem. Chem. Phys. 14, 11920 (2012).

[54] A. Karton, A. Tarnopolsky, J.-F. Lamère, G. C. Schatz abd Jan M. L. Martin, J. Phys. Chem. A 112, 12868 (2008).

[55] K. Sharkas, J. Toulouse and A. Savin, J. Chem. Phys. 134, 064113 (2011).

[56] E. Brémond and C. Adamo, J. Chem. Phys. 135, 024106 (2011).

[57] J. Toulouse, K. Sharkas, E. Brémond and C. Adamo, J. Chem. Phys. 135, 101102 (2011). 
[58] A. D. Becke, J. Chem. Phys. 98, 5648 (1993).

[59] J. P. Perdew, M. Ernzenhof and K. Burke, J. Chem. Phys. 105, 9982 (1996).

[60] C. Adamo and V. Barone, Chem. Phys. Lett. 274, 242 (1997).

[61] S. Grimme, J. Chem. Phys. 124, 034108 (2006).

[62] T. Schwabe and S. Grimme, Phys. Chem. Chem. Phys. 8, 4398 (2006).

[63] L. Goerigk and S. Grimme, J. Chem. Theory Comput. 7, 291 (2011).

[64] S. Grimme, J. Comput. Chem. 27, 1787 (2006).

[65] T. Schwabe and S. Grimme, Phys. Chem. Chem. Phys. 9, 3397 (2007).

[66] S. Grimme, J. Antony, S. Ehrlich and H. Krieg, J. Chem. Phys. 132, 154104 (2010).

[67] S. Grimme, WIREs Comput. Mol. Sci. 1, 211 (2011).

[68] O. A. Vydrov and T. Van Voorhis, J. Chem. Phys. 133, 244103 (2010).

[69] W. Hujo and S. Grimme, J. Chem. Theory Comput. 7, 3866 (2011).

[70] W. Hujo and S. Grimme, J. Chem. Theory Comput. 9, 308 (2013).

[71] J. Aragó, J. C. Sancho-García, and E. Ortí, submitted for publication.

[72] F. Neese, WIREs Comput. Mol. Sci. 2, 73 (2012).

[73] K. Eichkorn, O. Trutler, H. Öhm, M. Häser, and R. Ahlrichs, Chem. Phys. Lett. 240, 283 (1995).

[74] F. Neese, F. Wennmohs, A. Hansen, and U. Becker, Chem. Phys. 356, 98 (2009). 
[75] K. Eichkorn, F. Weigend, O. Trutler, and R. Ahlrichs, Theor. Chem. Acc. 97, 119 (1997).

[76] C. F. Macrae, I. J. Bruno, J. A. Chisholm, P. R. Edgington, P. McCabe, E. Pidcock, L. Rodríguez-Monge, R. Taylor, J. van de Streek, and P. A. Wood, J. Appl. Cryst. 41, 466 (2008).

[77] A. Otero-de-la-Roza and E. R. Johnson, J. Chem. Phys. 137, 054103 (2012).

[78] J. S. Chickos, Netsu Sokutei 30, 116 (2003).

[79] C. Pratt-Brock and J.D. Dunitz, Acta Crystallogr. Sect. B (Str.Sci.) 46, 795 (1990).

[80] A. L. Ringer and C. D. Sherrill, Chem. Eur. J. 14, 2542 (2008).

[81] A. Tkatchenko, R. A. DiStasio, R. Car, and M. Scheffler, Phys. Rev. Lett. 108, 236402 (2012).

[82] R. A. DiStasio, A. O. von Lilienfeld, and A. Tkatchenko, Proc. Natl. Acad. Sci. USA 109, 14791 (2012).

[83] A. Tkatchenko, A. Ambrosetti, and R. A. DiStasio, J. Chem. Phys. 138, 074106 (2013).

[84] W. B. Schweizer and J. B. Dunitz, J. Chem. Theory Comput. 2, 288 (2006).

[85] A. Halkier, T. Helgaker, P. Jorgensen, W. Klopper, H. Koch, J. Olsen, and A. K. Wilson, Chem. Phys. Lett. 286, 243 (1998).

[86] P. L. Fast, M. L. Sánchez, and D. G. Truhlar, J. Chem. Phys. 111, 2921 (1999). 
[87] O. A. von Lilienfeld and A. Tkatchenko, J. Chem. Phys. 132, 234109 (2010).

[88] Y. Liu and W. A. Goddard III, J. Phys. Chem. Lett. 1, 2550 (2010).

[89] W. A. Al-Saidi, V. K. Voora, and K. D. Jordan, J. Chem. Theory Comput. 8, 1503 (2012).

[90] I. A. Fedorov, Y. N. Zhuravlev, and V. P. Berveno, Phys. Chem. Chem. Phys. 13, 5679 (2011).

[91] Y. Zhang and W. Yang, Phys. Rev. Lett. 80, 890 (1998). 
- Table 1. List of parameters entering into the dispersion correction for all the methods employed.

- Table 2. Association energies (in $\mathrm{kJ} / \mathrm{mol}$ ) for interacting anthracene dimers in the first and second coordination sphere, and corresponding derived lattice or cohesive energies, at several theoretical levels. 
Table 1:

\begin{tabular}{lcccccc}
\hline Method & $s_{6}$ & $s_{r, 6}$ & $s_{8}$ & $s_{r, 8}$ & $b$ & Ref. \\
\hline B2-PLYP-D2 & 0.55 & 1.100 & - & - & - & {$[65]$} \\
mPW2-PLYP-D2 & 0.40 & 1.100 & - & - & - & {$[65]$} \\
B2-PLYP-D3 & 0.64 & 1.427 & 1.022 & 1.0 & - & {$[66]$} \\
PWPB95-D3 & 0.82 & 1.557 & 0.705 & 1.0 & - & {$[63]$} \\
B2-PLYP-NL & - & - & - & - & 8.3 & {$[71]$} \\
\hline
\end{tabular}


Table 2:

\begin{tabular}{|c|c|c|c|c|c|c|c|}
\hline & & $\mathrm{N}^{a}$ & $\begin{array}{l}\text { SCS-MP2 } \\
(\mathrm{CBS})\end{array}$ & $\begin{array}{c}\text { B2-PLYP } \\
\text { (def2-QZVP) }\end{array}$ & $\begin{array}{c}\text { B2-PLYP-D2 } \\
\text { (def2-QZVP) }\end{array}$ & $\begin{array}{l}\text { B2-PLYP-D3 } \\
\text { (def2-QZVP) }\end{array}$ & $\begin{array}{l}\text { B2-PLYP-NL } \\
\text { (def2-QZVP) }\end{array}$ \\
\hline \multicolumn{8}{|l|}{ 1st-shell } \\
\hline & $(\mathrm{a} / 2, \mathrm{~b} / 2,0)$ & 4 & -28.15 & -10.28 & -30.81 & -30.97 & -30.56 \\
\hline & $(\mathrm{a} / 2, \mathrm{~b} / 2, \mathrm{c})$ & 4 & -5.48 & -2.07 & -7.60 & -7.89 & -7.22 \\
\hline & $(-\mathrm{a} / 2, \mathrm{~b} / 2, \mathrm{c})$ & 4 & -0.59 & 0.07 & -0.04 & -0.07 & -0.11 \\
\hline & $(0,0, c)$ & 2 & -2.48 & -0.40 & -4.66 & -4.86 & -3.99 \\
\hline \multicolumn{8}{|l|}{ 2nd-shell } \\
\hline & $(\mathrm{a}, 0,0)$ & 2 & -0.82 & -0.29 & -1.12 & -1.31 & -1.64 \\
\hline & $(0, \mathrm{~b}, 0)$ & 2 & -16.82 & -8.68 & -21.22 & -22.08 & -21.23 \\
\hline & $(\mathrm{a}, \mathrm{b}, 0)$ & 4 & -0.88 & -0.26 & -0.66 & -0.76 & -0.88 \\
\hline & $(\mathrm{a}, 0, \mathrm{c})$ & 2 & -2.42 & -0.71 & -1.79 & -2.11 & -2.40 \\
\hline & $(-a, 0, c)$ & 2 & -0.49 & 0.13 & 0.11 & 0.11 & 0.10 \\
\hline & $(0, \mathrm{~b}, \mathrm{c})$ & 4 & -0.76 & -0.10 & -0.32 & -0.37 & -0.43 \\
\hline & $(\mathrm{a}, \mathrm{b}, \mathrm{c})$ & 4 & -0.49 & -0.13 & -0.18 & -0.26 & -0.34 \\
\hline & $(-a, b, c)$ & 4 & -0.46 & 0.19 & 0.18 & 0.17 & 0.17 \\
\hline Cohesive energy $^{b}$ & & & 96.68 & 34.58 & 107.58 & 110.54 & 107.91 \\
\hline
\end{tabular}

${ }^{a}$ Number of symmetry-related pairs.

${ }^{b}$ Note that the counting method needs dividing the total result by two (see Ref. [84]). 
- Figure 1. Chemical structure of anthracene.

- Figure 2. Interacting dimers (extracted from the crystalline structure) in the first and second coordination shell (from top to bottom and from left to right): (a/2,b/2,0); (a/2,b/2,c); (-a/2,b/2,c); (c,0,0); (a,0,0); $(0, b, 0) ;(a, b, 0) ;(a, 0, c) ;(-a, 0, c) ;(0, b, c) ;(a, b, c) ;$ and $(-a, b, c)$.

- Figure 3. Estimates of cohesive energies $(\mathrm{kJ} / \mathrm{mol})$ by different theoretical methods, with some B2-PLYP variants (with the the def2-QZVP basis set) and $a b$ initio methods (at the complete basis set limit). The experimental value (dashed red line) of $100.6 \mathrm{~kJ} / \mathrm{mol}$, and its average experimental uncertainty $( \pm 4.9 \mathrm{~kJ} / \mathrm{mol})$, are also included.

- Figure 4. Aggregate of anthracene molecules used to study 3-body interactions. 


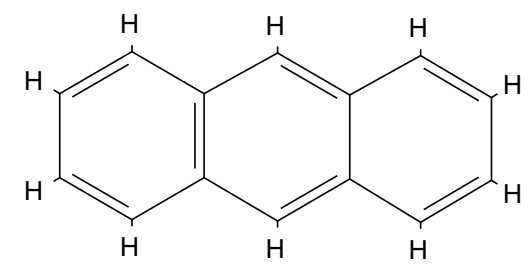

Figure 1. 

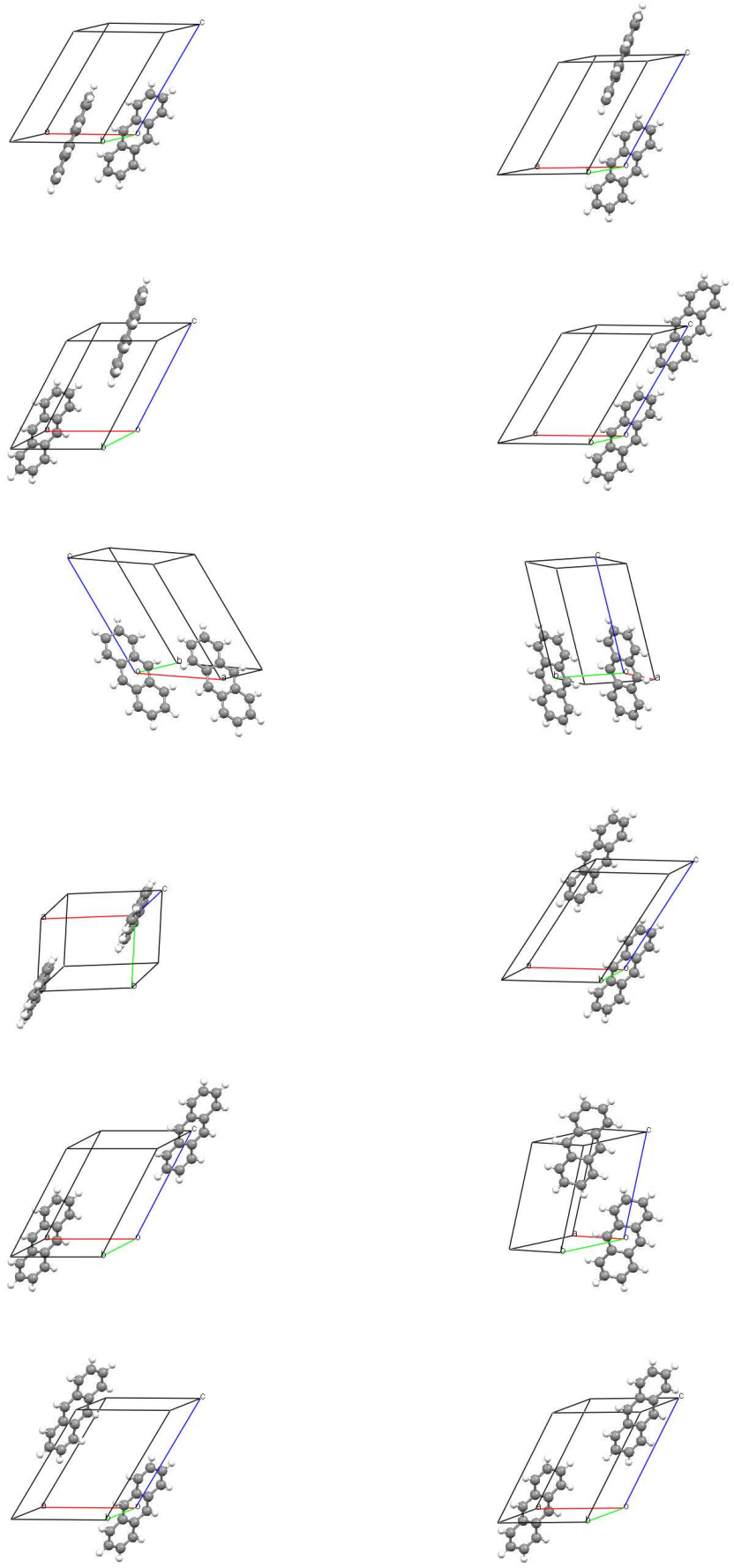

Figure 2. 


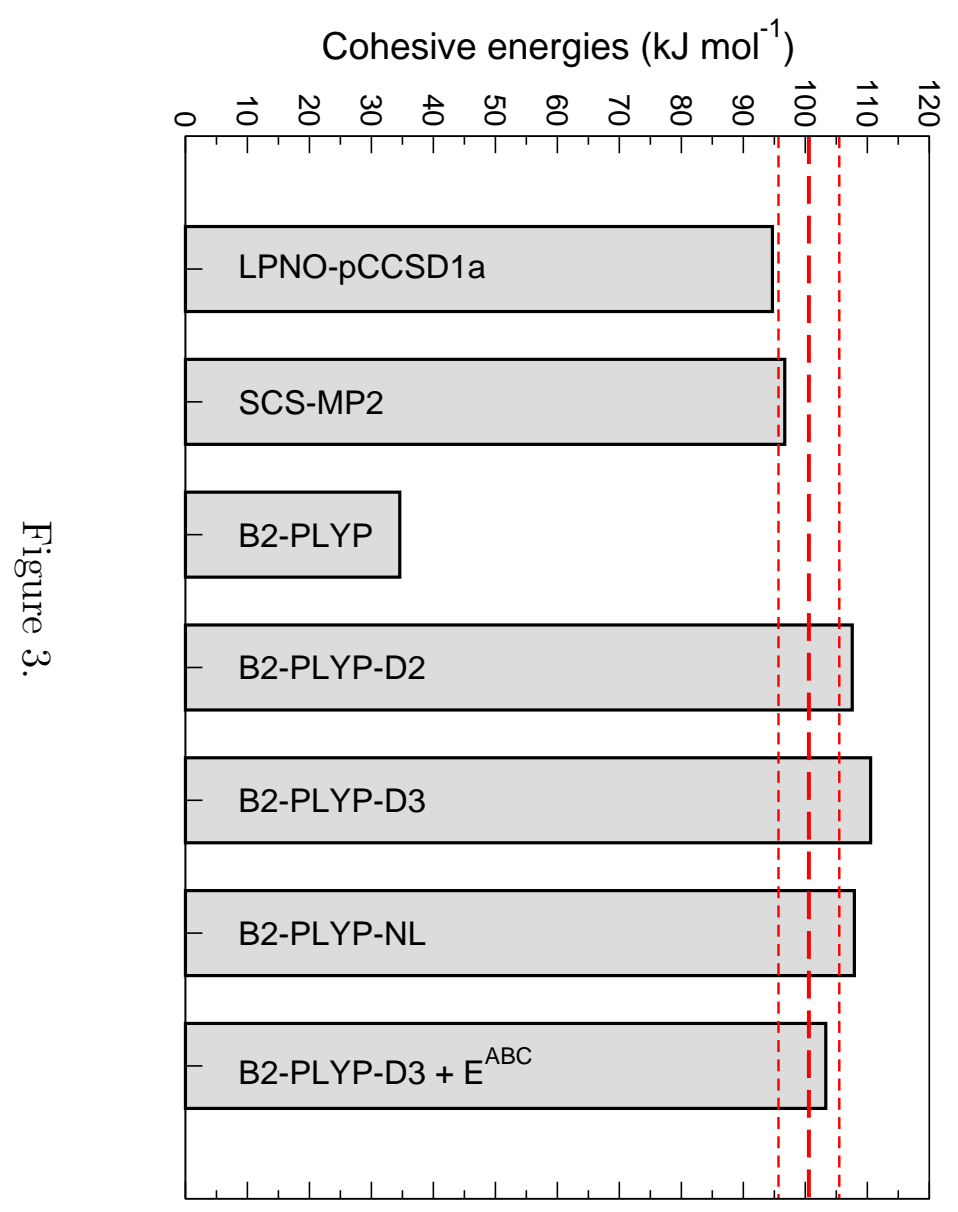




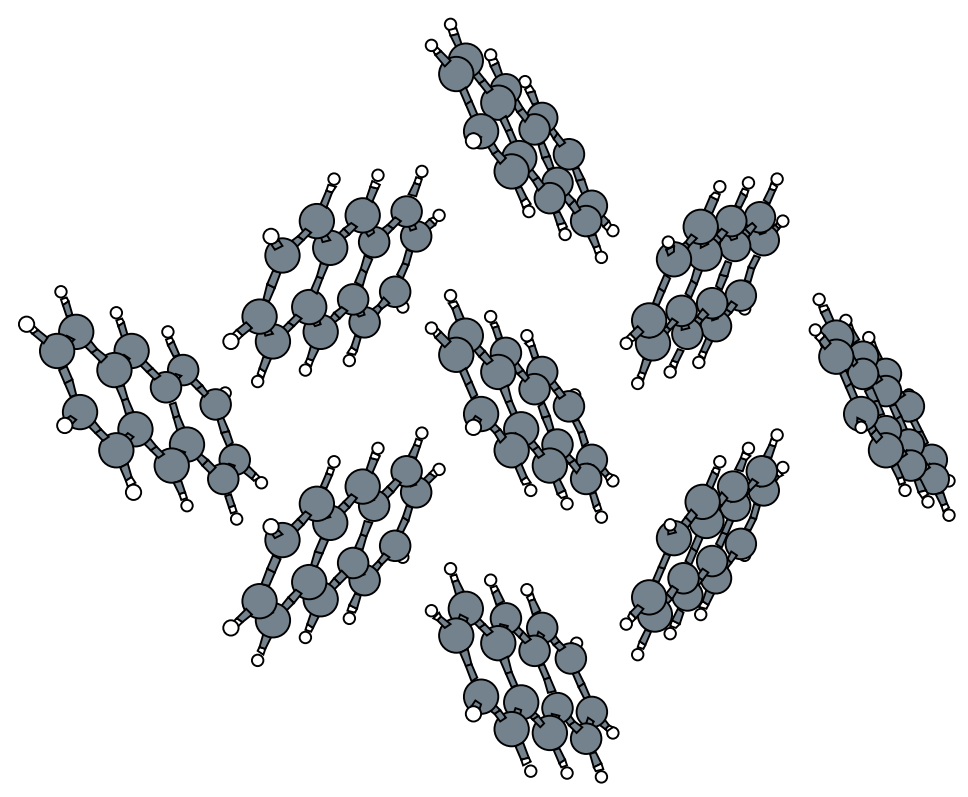

Figure 4. 